\title{
Numerical Approximation for Nonlinear Gas Dynamic Equation
}

\author{
Hossein Aminikhah and Ali Jamalian \\ Department of Applied Mathematics, School of Mathematical Sciences, University of Guilan, P.O. Box 41335-1914, Rasht, Iran \\ Correspondence should be addressed to Hossein Aminikhah; hossein.aminikhah@gmail.com
}

Received 15 March 2013; Revised 25 May 2013; Accepted 28 May 2013

Academic Editor: Zhenhua Guo

Copyright (C) $2013 \mathrm{H}$. Aminikhah and A. Jamalian. This is an open access article distributed under the Creative Commons Attribution License, which permits unrestricted use, distribution, and reproduction in any medium, provided the original work is properly cited.

Laplace transform and new homotopy perturbation methods are adopted to study gas dynamic equation analytically. The solutions introduced in this study can be used to obtain the closed form of the solutions if they are required. The combined method needs less work in comparison with the other homotopy perturbation methods and decreases volume of calculations considerably. Results show that the new method is more effective and convenient to use, and is high accuracy evident.

\section{Introduction}

Many phenomena in engineering, physics, chemistry, and other sciences can be described very successfully by nonlinear models. Except in a limited number of these problems, we have difficulty in finding their exact analytical solutions. Therefore, there have been attempts to develop new techniques for obtaining analytical solutions which reasonably approximate the exact solutions [1]. In recent decades, numerical calculation methods were good means of analyzing the nonlinear equations, but as the numerical calculation methods improved, analytical methods did, too. Most scientists believe that the combination of numerical and analytical methods can also end with useful results. In recent years, several of such techniques have drawn special attention, such as homotopy perturbation method. In the recent years, an increasing interest of scientists and engineers has been devoted to the analytical asymptotic techniques for solving nonlinear problems. Many new numerical techniques have been widely applied to the nonlinear problems. Based on homotopy, which is a basic concept in topology, general analytical method, namely, the homotopy perturbation method (HPM) is established by He [2-8] in 1998 to obtain series solutions of nonlinear differential equations. The He's HPM has been already used to solve various functional equations. In this method, the nonlinear problem is transferred to an infinite number of subproblems, and then the solution is approximated by the sum of the solutions of the first several subproblems. This simple method has been applied to solve linear and nonlinear equations of heat transfer [9-11], fluid mechanics [12], nonlinear Schrodinger equations [13], integral equations [14], boundary value problems [15], fractional KdV-Burgers equation [16], nonlinear system of secondorder boundary value problems [17], and delay differential equations [18].

The equations of gas dynamics are mathematical expressions based on the physical laws of conservation, namely, the laws of conservation of mass, conservation of momentum, conservation of energy, and so forth. The nonlinear equations of ideal gas dynamics are applicable for three types of nonlinear waves like shock fronts, rare factions, and contact discontinuities. Different types of gas dynamics equations in physics have been solved by Evans and Bulut [19], Polyanin and Zaitsev [20], Elizarova [21], Jafari et al. [22], and Ames [23], by applying various kinds of analytical and numerical methods. In 2003, Rasulov and Karaguler [24] applied the difference scheme for solving nonlinear system of equations of gas dynamic problems for a class of discontinuous functions.

In this work, we construct the solution of gas dynamic equation by using a powerful method. We obtain an analytical approximation to the solution of the nonlinear gas dynamic equation using combination of Laplace transform and new homotopy perturbation methods (LTNHPM). The 
gas dynamic equation as a nonlinear model is as follows $[19,22]$ :

$$
u_{t}+u u_{x}-u(1-u)=0
$$

where $0 \leq x \leq 1$ and $t>0$. The results obtained via LTNHPM confirm validity of the proposed method. The rest of this paper is organized as follows.

In Section 2, basic ideas of LTNHPM and the homotopy perturbation method are presented. In Section 3, the application of LTNHPM for solving nonlinear gas dynamic equation is presented. Conclusion will appear in the last section.

\section{Basic Ideas of the LTNHPM}

In this section, we propose LTNHPM, a new hybrid of Laplace transform and new homotopy perturbation methods (NHPM) for solving nonlinear gas dynamic equation. This method is simple and finds exact solution of the equations analytically using the initial condition only. This method unlike the most numerical techniques provides a closed form of the solution. The new method developed in the current paper was tested on several equations [25-27]. To illustrate the basic ideas of this method, let us consider the following nonlinear differential equation:

$$
A(u)-f(r)=0, \quad r \in \Omega,
$$

with the following initial conditions:

$$
u(0)=\alpha_{0}, \quad u^{\prime}(0)=\alpha_{1}, \ldots, u^{(n-1)}(0)=\alpha_{n-1},
$$

where $A$ is a general differential operator and $f(r)$ is a known analytical function. The operator $A$ can be divided into two parts, $L$ and $N$, where $L$ is a linear and $N$ is a nonlinear operator. Therefore, (2) can be rewritten as

$$
L(u)+N(u)-f(r)=0 .
$$

Based on NHPM [28], we construct a homotopy $U(r, p): \Omega \times$ $[0,1] \rightarrow \mathbb{R}$, which satisfies

$$
\begin{aligned}
H(U, p)= & (1-p)\left[L(U)-u_{0}\right] \\
& +p[A(U)-f(r)] \\
= & 0, \quad p \in[0,1], r \in \Omega,
\end{aligned}
$$

or equivalently,

$$
H(U, p)=L(U)-u_{0}+p u_{0}+p[N(U)-f(r)]=0,
$$

where $p \in[0,1]$ is an embedding parameter and $u_{0}$ is an initial approximation for the solution of (2). Clearly, (5) and (6) give

$$
\begin{gathered}
H(U, 0)=L(U)-u_{0}=0, \\
H(U(x), 1)=A(U)-f(r)=0 .
\end{gathered}
$$

Applying Laplace transform to both sides of (6), we arrive at

$$
L\left\{L(U)-u_{0}+p u_{0}+p[N(U)-f(r)]\right\}=0 .
$$

Using the differential property of Laplace transform we have

$$
\begin{gathered}
s^{n} L\{U\}-s^{n-1} U(0)-s^{n-2} U^{\prime}(0)-\cdots-U^{(n-1)}(0) \\
=L\left\{u_{0}-p u_{0}+p[N(U)-f(r)]\right\}
\end{gathered}
$$

or

$$
\begin{array}{r}
L\{U\}=\frac{1}{s^{n}}\left\{s^{n-1} U(0)+s^{n-2} U^{\prime}(0)+\cdots+U^{(n-1)}(0)\right. \\
\left.+L\left\{u_{0}-p u_{0}+p[N(U)-f(r)]\right\}\right\} .
\end{array}
$$

Finally, applying the inverse Laplace transform to both sides of (10), one can successfully reach the following:

$$
\begin{array}{r}
U=L^{-1}\left\{\frac { 1 } { s ^ { n } } \left\{s^{n-1} U(0)+s^{n-2} U^{\prime}(0)+\cdots+U^{(n-1)}(0)\right.\right. \\
\left.\left.+L\left\{u_{0}-p u_{0}+p[N(U)-f(r)]\right\}\right\}\right\} .
\end{array}
$$

According to the HPM, we can first use the embedding parameter $p$ as a small parameter and assume that the solutions of (11) can be represented as a power series in $p$ as

$$
U(x)=\sum_{n=0}^{\infty} p^{n} U_{n} .
$$

Now let us rewrite (11) using (12) as

$$
\begin{aligned}
& \sum_{n=0}^{\infty} p^{n} U_{n} \\
&=L^{-1}\left\{\frac { 1 } { s ^ { n } } \left\{s^{n-1} U(0)+s^{n-2} U^{\prime}(0)+\cdots+U^{(n-1)}(0)\right.\right. \\
&+L\left\{u_{0}-p u_{0}+p\left[N\left(\sum_{n=0}^{\infty} p^{n} U_{n}\right)\right.\right. \\
&-f(r)]\}\}\} .
\end{aligned}
$$


Therefore, equating the coefficients of $p$ with the same power leads to

$$
\begin{aligned}
& p^{0}: U_{0}=L^{-1}\left\{\frac { 1 } { s ^ { n } } \left(s^{n-1} U(0)+s^{n-2} U^{\prime}(0)\right.\right. \\
& \left.\left.+\cdots+U^{(n-1)}(0)+L\left\{u_{0}\right\}\right)\right\} \\
& p^{1}: U_{1}=L^{-1}\left\{\frac{1}{s^{n}}\left(L\left\{N\left(U_{0}\right)-u_{0}-f(r)\right\}\right)\right\}, \\
& p^{2}: U_{2}=L^{-1}\left\{\frac{1}{s^{n}}\left(L\left\{N\left(U_{0}, U_{1}\right)\right\}\right)\right\} \text {, } \\
& p^{3}: U_{3}=L^{-1}\left\{\frac{1}{s^{n}}\left(L\left\{N\left(U_{0}, U_{1}, U_{2}\right)\right\}\right)\right\} \text {, } \\
& p^{j}: U_{j}=L^{-1}\left\{\frac{1}{s^{n}}\left(L\left\{N\left(U_{0}, U_{1}, U_{2}, \ldots, U_{j-1}\right)\right\}\right)\right\}, \\
& \vdots
\end{aligned}
$$

Suppose that the initial approximation has the form $U(0)=$ $u_{0}=\alpha_{0}, U^{\prime}(0)=\alpha_{1}, \ldots, U^{(n-1)}(0)=\alpha_{n-1}$, therefore the exact solution may be obtained as following

$$
u=\lim _{p \rightarrow 1} U=U_{0}+U_{1}+U_{2}+\cdots
$$

To show the capability of the method, we apply the NHPM to some examples in Section 3.

\section{Analysis of the Method}

Example 1. Consider the nonlinear gas dynamic equation (1)

$$
u_{t}+u u_{x}-u(1-u)=0
$$

subject to a constant initial condition

$$
u(x, 0)=a e^{-x} .
$$

For solving this equation by applying the new homotopy perturbation method, we construct the following homotopy:

$$
H(U, p)=U_{t}-u_{0}+p\left[u_{0}+U U_{x}-U(1-U)\right]=0,
$$

where $p \in[0,1]$ is an embedding parameter, $u_{0}$ is an initial approximation of solution of equation. Clearly, we have from (18)

$$
\begin{gathered}
H(U, 0)=U_{t}-u_{0}=0 \\
H(U, 1)=U_{t}+U U_{x}-U(1-U)=0 .
\end{gathered}
$$

By applying Laplace transform on both sides of (18), we have

$$
L\{H(U, p)\}=L\left\{U_{t}-u_{0}+p\left[u_{0}+U U_{x}-U(1-U)\right]\right\} .
$$

Using the differential property of Laplace transform we have:

$$
\begin{aligned}
& s L\{U(x, t)\}-U(x, 0) \\
& \quad=L\left\{u_{0}-p\left[u_{0}+U U_{x}-U(1-U)\right]\right\}
\end{aligned}
$$

or

$$
\begin{aligned}
& L\{U(x, t)\} \\
& \quad=\frac{1}{s}\left(U(x, 0)+L\left\{u_{0}-p\left[u_{0}+U U_{x}-U(1-U)\right]\right\}\right) .
\end{aligned}
$$

By applying inverse Laplace transform on both sides of (22), we have

$U(x, t)$

$$
=L^{-1}\left\{\frac{1}{s}\left(U(x, 0)+L\left\{u_{0}-p\left[u_{0}+U U_{x}-U(1-U)\right]\right\}\right)\right\} .
$$

According to the HPM, we use the embedding parameter $p$ as a small parameter, and assume that the solutions of (23) can be represented as a power series in $p$ as

$$
U(x, t)=\sum_{n=0}^{\infty} p^{n} U_{n}(x, t) .
$$

Substituting (24) into (23), and equating the terms with the identical powers of $p$, leads to calculate $U_{j}(x, t), j=0,1,2, \ldots$

$$
\begin{aligned}
p^{0}: & U_{0}(x, t)=L^{-1}\left\{\frac{1}{s}\left(U(x, 0)+L\left\{u_{0}(x, t)\right\}\right)\right\} \\
p^{1}: & U_{1}(x, t) \\
& =L^{-1}\left\{\frac{-1}{s} L\left\{u_{0}(x, t)+U_{0}\left(U_{0}\right)_{x}-U_{0}+U_{0}^{2}\right\}\right\} \\
p^{2}: & U_{2}(x, t) \\
& =L^{-1}\left\{\frac{-1}{s} L\left\{U_{0}\left(U_{1}\right)_{x}+U_{1}\left(U_{0}\right)_{x}-U_{1}+2 U_{0} U_{1}\right\}\right\}
\end{aligned}
$$$$
p^{j}: U_{j}(x, t)
$$$$
=L^{-1}\left\{\frac { - 1 } { s } L \left\{\sum_{k=0}^{j-1} U_{k}\left(U_{j-k-1}\right)_{x}-U_{j-1}\right.\right.
$$$$
\left.\left.+\sum_{k=0}^{j-1} U_{k} U_{j-k-1}\right\}\right\} .
$$

Suppose that the initial approximation has the form $U(x, 0)=$ $u_{0}(x, t)=a e^{-x}$, therefore the exact solution may be obtained as following

$$
\begin{aligned}
u(x, t) & =\lim _{p \rightarrow 1} U(x, t) \\
& =U_{0}(x, t)+U_{1}(x, t)+U_{2}(x, t)+\cdots .
\end{aligned}
$$


Solving (25) for $U_{j}(x, t), j=0,1, \ldots$ leads to the result

$$
\begin{aligned}
& U_{0}(x, t)=e^{-x}(1+t), \\
& U_{1}(x, t)=\frac{1}{2} a e^{-x} t^{2}, \\
& U_{2}(x, t)=\frac{1}{6} a e^{-x} t^{3}, \\
& U_{3}(x, t)=\frac{1}{24} a e^{-x} t^{4} \\
& U_{4}(x, t)=\frac{1}{120} a e^{-x} t^{5}, \\
& \vdots
\end{aligned}
$$

Therefore, we gain the solution of (27) as

$$
\begin{aligned}
u(x, t)= & U_{0}(x, t)+U_{1}(x, t)+U_{2}(x, t) \\
& +U_{3}+(x, t)+\cdots \\
= & a e^{-x}+a e^{-x} t+a e^{-x} \frac{t^{2}}{2 !}+a e^{-x} \frac{t^{3}}{3 !} \\
& +a e^{-x} \frac{t^{4}}{4 !}+a e^{-x} \frac{t^{5}}{5 !}+\cdots=a e^{t-x},
\end{aligned}
$$

which is the exact solution of the problem. Figure 1 shows the plot of $u(x, t)=a e^{t-x}$ with $a=1$ and $t>0,0 \leq x \leq 1$.

Example 2. Consider the following nonhomogeneous nonlinear gas dynamic equation [19]:

$$
u_{t}+u u_{x}-u(1-u)=-e^{t-x}
$$

with the initial condition

$$
u(x, 0)=1-e^{-x} .
$$

For solving this equation by applying the new homotopy perturbation method, we construct the following homotopy:

$$
\begin{aligned}
H & (U, p) \\
& =U_{t}-u_{0}+p\left[u_{0}+U U_{x}-U(1-U)+e^{t-x}\right] \\
& =0,
\end{aligned}
$$

where $p \in[0,1]$ is an embedding parameter, $u_{0}$ is an initial approximation of solution of equation. Clearly, we have from (31)

$$
\begin{gathered}
H(U, 0)=U_{t}-u_{0}=0, \\
H(U, 1)=U_{t}+U U_{x}-U(1-U)+e^{t-x}=0 .
\end{gathered}
$$

By applying Laplace transform on both sides of (31), we have

$$
\begin{aligned}
& L\{H(U, p)\} \\
& \quad=L\left\{U_{t}-u_{0}+p\left[u_{0}+U U_{x}-U(1-U)+e^{t-x}\right]\right\} .
\end{aligned}
$$

Using the differential property of Laplace transform we have

$$
\begin{aligned}
& \quad s L\{U(x, t)\}-U(x, 0) \\
& \quad=L\left\{u_{0}-p\left[u_{0}+U U_{x}-U(1-U)+e^{t-x}\right]\right\} \\
& L\{U(x, t)\} \\
& =\frac{1}{s}(U(x, 0) \\
& \left.\quad+L\left\{u_{0}-p\left[u_{0}+U U_{x}-U(1-U)+e^{t-x}\right]\right\}\right) .
\end{aligned}
$$

or

By applying inverse Laplace transform on both sides of (35), we have

$$
\begin{aligned}
& U(x, t) \\
& =L^{-1}\left\{\frac{1}{s}(U(x, 0)\right. \\
& \left.\left.\quad+L\left\{u_{0}-p\left[u_{0}+U U_{x}-U(1-U)+e^{t-x}\right]\right\}\right)\right\} .
\end{aligned}
$$

According to the HPM, we use the embedding parameter $p$ as a small parameter and assume that the solutions of (36) can be represented as a power series in $p$ as

$$
U(x, t)=\sum_{n=0}^{\infty} p^{n} U_{n}(x, t) .
$$

Substituting (37) into (36) and equating the terms with the identical powers of $p$ lead to calculating $U_{j}(x, t), j=$ $0,1,2, \ldots$

$$
\begin{aligned}
p^{0}: & U_{0}(x, t)=L^{-1}\left\{\frac{1}{s}\left(U(x, 0)+L\left\{u_{0}(x, t)\right\}\right)\right\}, \\
p^{1}: & U_{1}(x, t) \\
& =L^{-1}\left\{\frac{-1}{s} L\left\{u_{0}(x, t)+U_{0}\left(U_{0}\right)_{x}-U_{0}+U_{0}^{2}+e^{t-x}\right\}\right\}, \\
p^{2}: & U_{2}(x, t) \\
& =L^{-1}\left\{\frac{-1}{s} L\left\{U_{0}\left(U_{1}\right)_{x}+U_{1}\left(U_{0}\right)_{x}-U_{1}+2 U_{0} U_{1}\right\}\right\}, \\
\vdots & p^{j}: \quad U_{j}(x, t) \\
& =L^{-1}\left\{\frac{-1}{s} L\left\{\sum_{k=0}^{j-1} U_{k}\left(U_{j-k-1}\right)_{x}-U_{j-1}+\sum_{k=0}^{j-1} U_{k} U_{j-k-1}\right\}\right\} .
\end{aligned}
$$

Suppose that the initial approximation has the form $U(x, 0)=$ $u_{0}(x, t)=1-e^{-x}$; therefore, the exact solution may be obtained as follows

$$
\begin{aligned}
u(x, t) & =\lim _{p \rightarrow 1} U(x, t) \\
& =U_{0}(x, t)+U_{1}(x, t)+U_{2}(x, t)+\cdots
\end{aligned}
$$




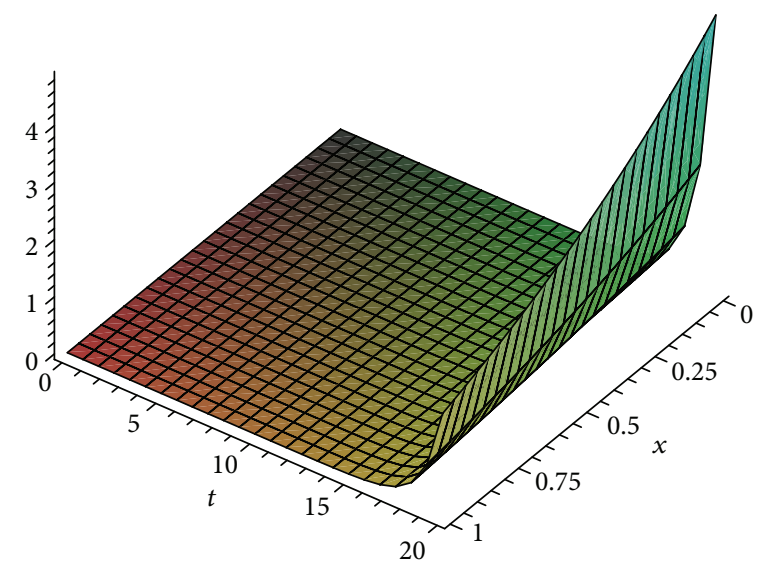

FIGURE 1: Plot of $u(x, t)=a e^{t-x}$ with $a=1$ and $t>0,0 \leq x \leq 1$.

Solving (38) for $U_{j}(x, t), j=0,1,2, \ldots$ leads to the result

$$
\begin{aligned}
U_{0}(x, t)=1 & +t-e^{-x}(1+t), \\
U_{1}(x, t)=- & t-\frac{t^{2}}{2}-\frac{t^{3}}{3}-e^{t-x} \\
& +\frac{1}{6} e^{-x}\left(2 t^{3}+3 t^{2}+6 t+6\right), \\
U_{2}(x, t)= & \frac{t^{2}}{2}+\frac{5}{6} t^{3}+\frac{t^{4}}{3}+\frac{2}{15} t^{5} \\
& -\frac{1}{30} e^{-x}\left(10 t^{4}+4 t^{5}-30+30 t^{2}+25 t^{3}\right) \\
& +e^{t-x}(t-1), \\
U_{3}(x, t)= & -\frac{t^{3}}{2}-\frac{17}{90} t^{6}-\frac{17}{24} t^{4}-\frac{7}{12} t^{5}-\frac{17}{315} t^{7} \\
& -\frac{1}{6} e^{t-x}\left(2 t^{3}+3 t^{2}-6 t+6\right) \\
& +\frac{1}{1260} e^{-x}\left(68 t^{7}+1155 t^{4}+238 t^{6}\right. \\
& +\frac{1}{45360} e^{-x}\left(16272 t^{7}+4464 t^{8}+28350 t^{4}\right. \\
& \left.+\frac{3}{8} t^{4}+\frac{31}{40} t^{5}+\frac{62}{2835} t^{9}+1260+840 t^{3}\right), \\
U_{4}(x, t)= & \frac{31}{315} t^{8}+\frac{23}{36} t^{6}+\frac{113}{315} t^{7} \\
& \left.+992 t^{9}-45360\right) \\
&
\end{aligned}
$$

$$
\begin{gathered}
+\frac{1}{30} e^{t-x}\left(-15 t^{2}+5 t^{3}+4 t^{5}\right. \\
\left.+30 t+10 t^{4}-30\right) \\
U_{5}(x, t)=-\frac{578}{2835} t^{9}-\frac{148}{315} t^{8}-\frac{57}{80} t^{6}-\frac{31}{40} t^{7} \\
-\frac{691}{14175} t^{10}-\frac{13}{40} t^{5}-\frac{1382}{155925} t^{11} \\
+\frac{1}{19958400} e^{-x}\left(17364600 t^{7}+10062855 t^{8}\right. \\
\quad+176896 t^{11}+18905040 t^{6} \\
+9646560 t^{5}+4069120 t^{9} \\
\left.+19958400+972928 t^{10}\right) \\
-\frac{1}{2520} e^{t-x}\left(105 t^{4}-420 t^{3}+798 t^{5}\right. \\
-2520 t+476 t^{6} \\
\left.+1260 t^{2}+136 t^{7}+2520\right)
\end{gathered}
$$

Therefore, we gain the solution of (40) as follows:

$$
\begin{aligned}
u(x, t) \cong & U_{0}(x, t)+U_{1}(x, t)+U_{2}(x, t)+U_{3} \\
& +(x, t)+U_{4}+(x, t)+U_{5}+(x, t) \\
= & 1+4 e^{-x}-5 e^{t-x}-\frac{13}{35} t^{8}-\frac{172}{945} t^{9} \\
& -\frac{11}{60} e^{t-x} t^{5}+\frac{7}{24} e^{t-x} t^{4}+\frac{16361}{40320} e^{-x} t^{8} \\
& +\frac{172}{945} e^{-x} t^{9}-\frac{17}{90} e^{t-x} t^{6}-\frac{17}{315} e^{t-x} t^{7}
\end{aligned}
$$




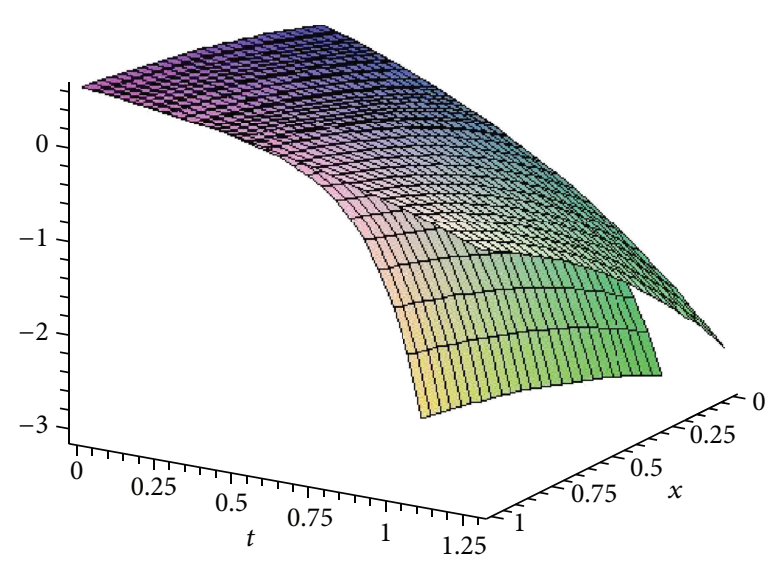

FIGURE 2: Plot of $u(x, t)=1-e^{t-x}$ with $t>0,0 \leq x \leq 1$, and the 5-term approximation of $u(x, t)$.

$$
\begin{aligned}
& +\frac{1382}{155925} e^{-x} t^{11}+\frac{691}{14175} e^{-x} t^{10} \\
& -\frac{21}{80} t^{6}-\frac{79}{168} t^{7}-\frac{3}{2} e^{t-x} t^{2} \\
& +4 e^{t-x} t+\frac{407}{720} e^{-x} t^{7}-\frac{1}{24} e^{-x} t^{4} \\
& +\frac{33}{80} e^{-x} t^{6}+\frac{1}{120} e^{-x} t^{5}+\frac{1}{6} e^{-x} t^{3} \\
& -\frac{691}{14175} t^{10}-\frac{1382}{155925} t^{11}-\frac{1}{2} e^{-x} t^{2}
\end{aligned}
$$

which is the exact solution of the problem. Figure 2 shows the plots of $u(x, t)=1-e^{t-x}$ with $t>0,0 \leq x \leq 1$, and the 5 -term approximation of $u(x, t)$.

\section{Summary and Conclusion}

In the present work, we proposed a combination of Laplace transform method and homotopy perturbation method to solve nonlinear gas dynamic equation. This method finds an exact solution of the equation using the initial condition only. This method unlike the most numerical techniques provides a closed form of the solution. The obtained results show that the new method developed in the current paper can solve the problem effectively.

\section{References}

[1] A. H. Nayfeh, Introduction to Perturbation Techniques, WileyInterscience, New York, NY, USA, 1981.

[2] J.-H. He, "Homotopy perturbation technique," Computer Methods in Applied Mechanics and Engineering, vol. 178, no. 3-4, pp. 257-262, 1999.

[3] J.-H. He, "Coupling method of a homotopy technique and a perturbation technique for non-linear problems," International Journal of Non-Linear Mechanics, vol. 35, no. 1, pp. 37-43, 2000.

[4] J.-H. He, "Addendum: new interpretation of homotopy perturbation method," International Journal of Modern Physics B, vol. 20, no. 18, pp. 2561-2568, 2006.
[5] J.-H. He, "Recent development of the homotopy perturbation method," Topological Methods in Nonlinear Analysis, vol. 31, no. 2, pp. 205-209, 2008.

[6] J.-H. He, "The homotopy perturbation method for nonlinear oscillators with discontinuities," Applied Mathematics and Computation, vol. 151, no. 1, pp. 287-292, 2004.

[7] J.-H. He, "Application of homotopy perturbation method to nonlinear wave equations," Chaos, Solitons and Fractals, vol. 26, no. 3, pp. 695-700, 2005.

[8] J.-H. He, "Limit cycle and bifurcation of nonlinear problems," Chaos, Solitons and Fractals, vol. 26, no. 3, pp. 827-833, 2005.

[9] A. Rajabi, D. D. Ganji, and H. Taherian, "Application of homotopy perturbation method in nonlinear heat conduction and convection equations," Physics Letters A, vol. 360, no. 4-5, pp. 570-573, 2007.

[10] D. D. Ganji and A. Sadighi, "Application of homotopyperturbation and variational iteration methods to nonlinear heat transfer and porous media equations," Journal of Computational and Applied Mathematics, vol. 207, no. 1, pp. 24-34, 2007.

[11] D. D. Ganji, "The application of He's homotopy perturbation method to nonlinear equations arising in heat transfer," Physics Letters A, vol. 355, no. 4-5, pp. 337-341, 2006.

[12] S. Abbasbandy, "A numerical solution of Blasius equation by Adomian's decomposition method and comparison with homotopy perturbation method," Chaos, Solitons and Fractals, vol. 31, no. 1, pp. 257-260, 2007.

[13] J. Biazar and H. Ghazvini, "Exact solutions for non-linear Schrödinger equations by He's homotopy perturbation method," Physics Letters A, vol. 366, no. 1-2, pp. 79-84, 2007.

[14] S. Abbasbandy, "Numerical solutions of the integral equations: homotopy perturbation method and Adomian's decomposition method," Applied Mathematics and Computation, vol. 173, no. 1, pp. 493-500, 2006.

[15] J.-H. He, "Homotopy perturbation method for solving boundary value problems," Physics Letters A, vol. 350, no. 1-2, pp. 8788, 2006.

[16] Q. Wang, "Homotopy perturbation method for fractional KdVBurgers equation," Chaos, Solitons and Fractals, vol. 35, no. 5, pp. 843-850, 2008.

[17] E. Yusufoğlu, "Homotopy perturbation method for solving a nonlinear system of second order boundary value problems," International Journal of Nonlinear Sciences and Numerical Simulation, vol. 8, no. 3, pp. 353-358, 2007. 
[18] J. Biazar and B. Ghanbari, "The homotopy perturbation method for solving neutral functional-differential equations with proportional delays," Journal of King Saud University, vol. 24, no. 1, pp. 33-37, 2012.

[19] D. J. Evans and H. Bulut, "A new approach to the gas dynamics equation: an application of the decomposition method," International Journal of Computer Mathematics, vol. 79, no. 7, pp. 817822, 2002.

[20] A. D. Polyanin and V. F. Zaitsev, Handbook of Nonlinear Partial Differential Equations, Chapman \& Hall/CRC, Boca Raton, Fla, USA, 2004.

[21] T. G. Elizarova, Quasi-Gas Dynamic Equations, Computational Fluid and Solid Mechanics, Springer, Dordrecht, The Netherlands, 2009.

[22] H. Jafari, C. Chun, S. Seifi, and M. Saeidy, "Analytical solution for nonlinear gas dynamic equation by homotopy analysis method," Applications and Applied Mathematics, vol. 4, no. 1, pp. 149-154, 2009.

[23] W. F. Ames, Nonlinear Partial Differential Equations in Engineering, Academic Press, New York, NY, USA, 1965.

[24] M. Rasulov and T. Karaguler, "Finite difference schemes for solving system equations of gas dynamic in a class of discontinuous functions," Applied Mathematics and Computation, vol. 143, no. 1, pp. 145-164, 2003.

[25] H. Aminikhah and A. Jamalian, "An analytical approximation for boundary layer flow convection heat and mass transfer over a flat plate," The Journal of Mathematics and Computer Science, vol. 5, no. 4, pp. 241-257, 2012.

[26] H. Aminikhah, F. Mehrdoust, and A. Jamalian, "A new efficient method for nonlinear Fisher-type equations," Journal of Applied Mathematics, vol. 2012, Article ID 586454, 18 pages, 2012.

[27] H. Aminikhah and A. Jamalian, "A new efficient method for solving the nonlinear Fokker-Planck equation," Scientia Iranica, vol. 19, pp. 1133-1139, 2012.

[28] H. Aminikhah and M. Hemmatnezhad, "An efficient method for quadratic Riccati differential equation," Communications in Nonlinear Science and Numerical Simulation, vol. 15, no. 4, pp. 835-839, 2010. 


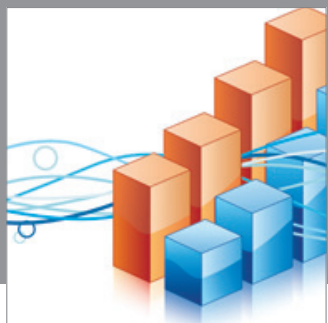

Advances in

Operations Research

mansans

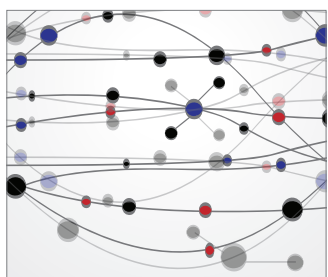

The Scientific World Journal
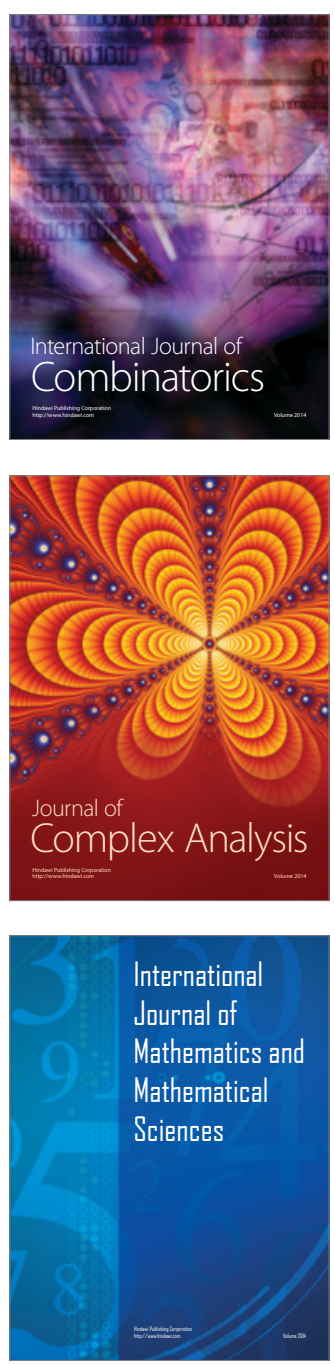
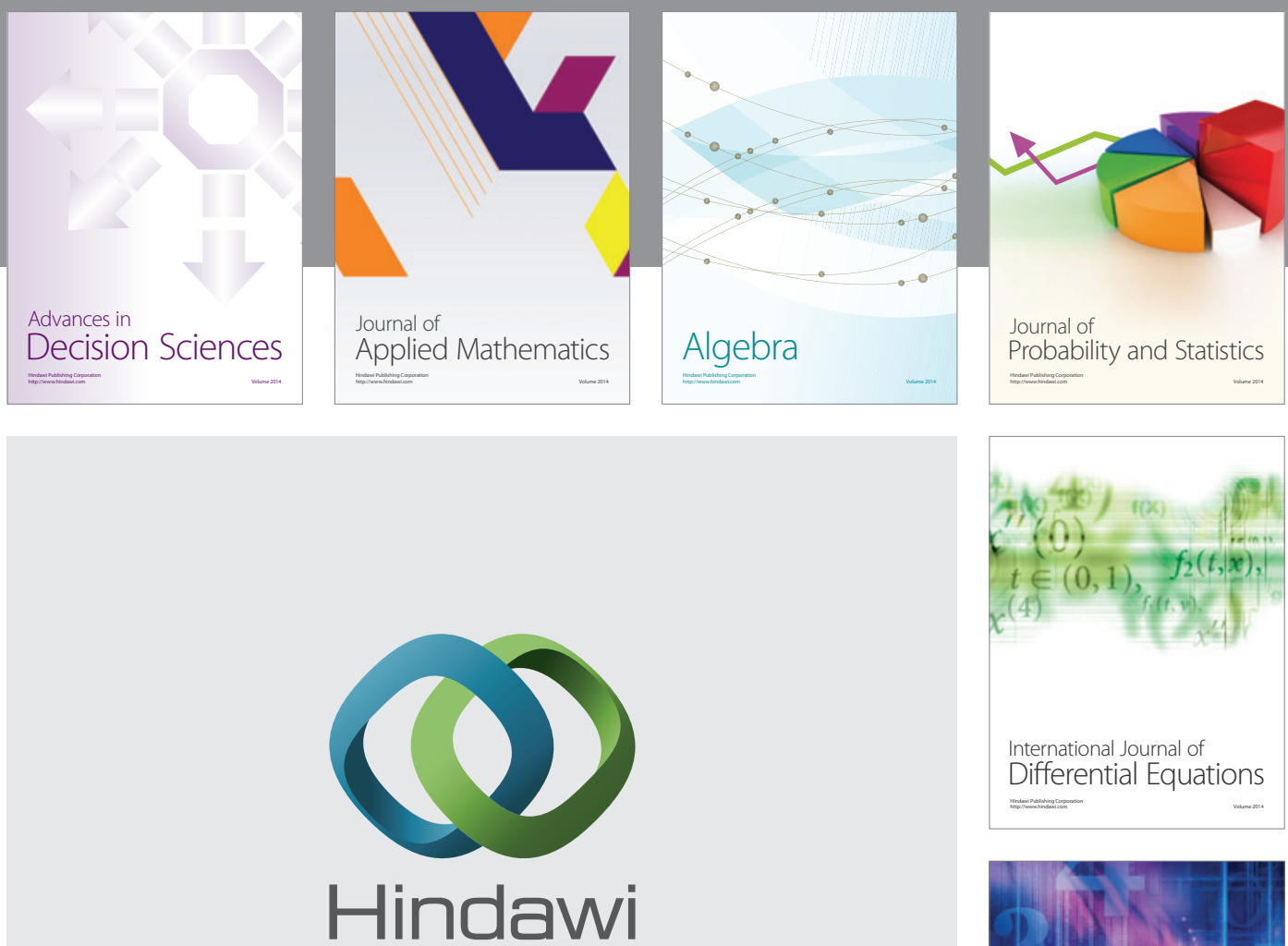

Submit your manuscripts at http://www.hindawi.com
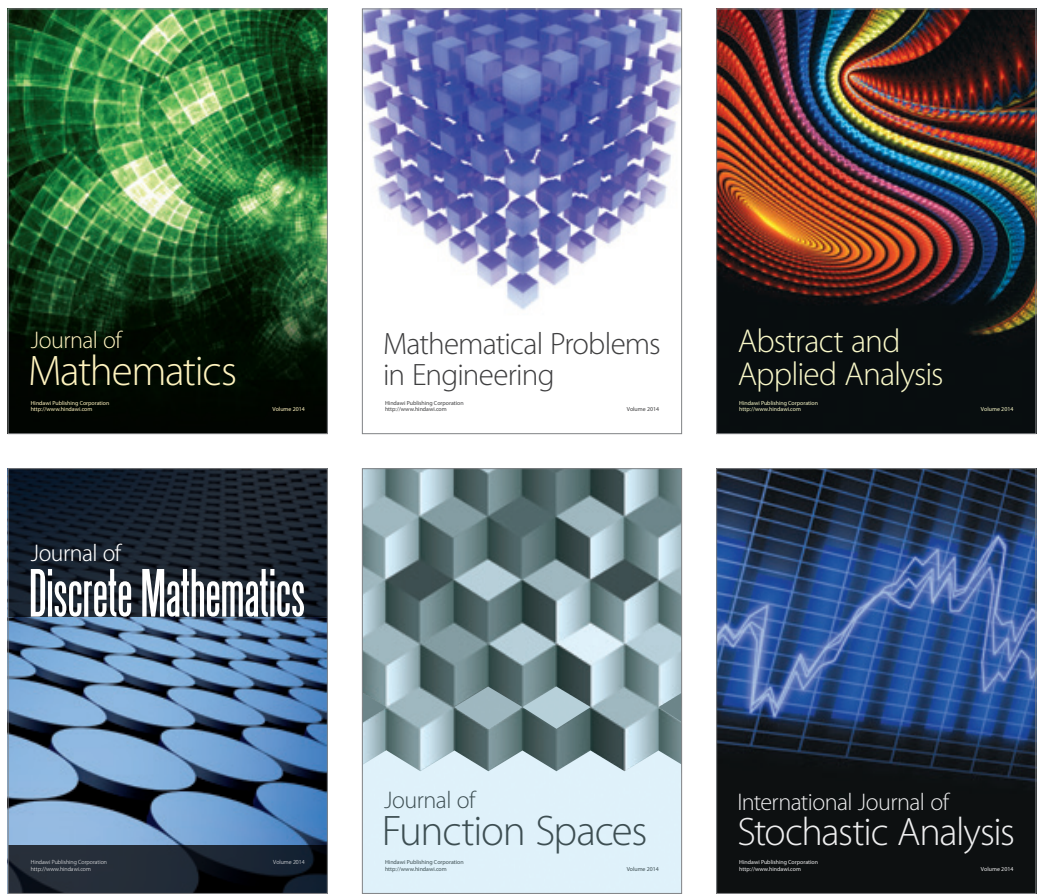

Journal of

Function Spaces

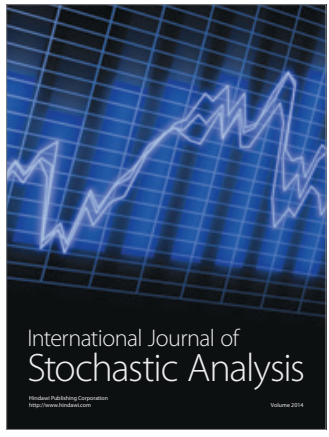

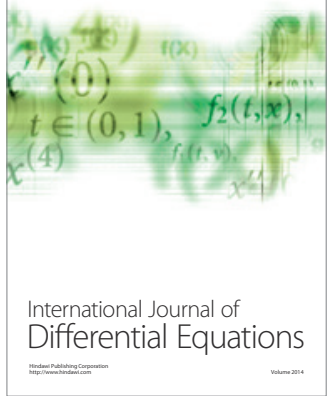
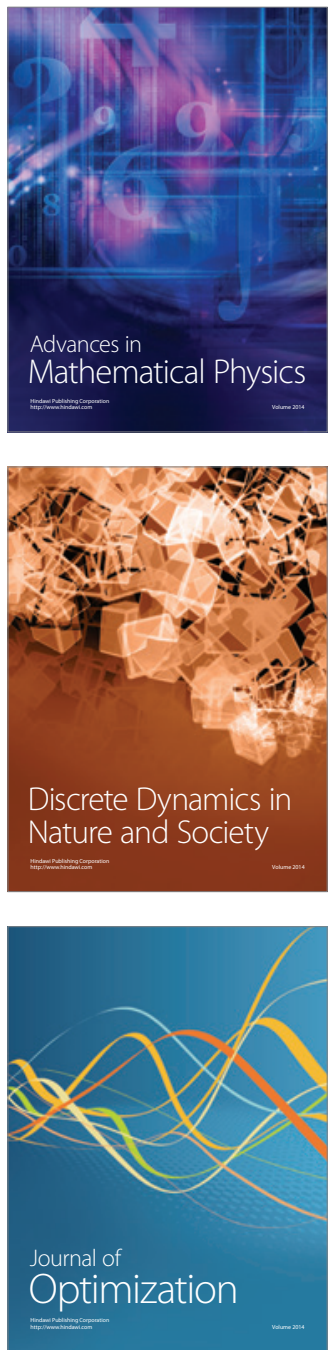